\title{
AN ANALYSIS OF SOME GENERAL PROPOSITIONS*
}

\author{
BY C. H. LANGFORD
}

1. Introduction. General propositions are commonly described as being those propositions which arise from matrices by generalization, that is, as being such propositions as can be derived from some matrix by the attachment of an applicative, "some" or "every," to each variable constituent of the matrix. $\dagger$ In this paper an analysis of general propositions is suggested, which results from a slightly different view of the relations of general propositions to matrices. It appears that the analysis which is suggested involves a generalization of the ordinary analysis.

2. Unanalysed Propositions. We may begin with a consideration of elementary matrices whose values are elementary functions of unanalysed propositions, such as, for example, $p \supset q$. Let $t_{0}$ denote any elementary proposition. Then we can write, for example, $\left(t_{0}\right) \cdot t_{0} \vee \sim t_{0}$, that is, every elementary proposition is true or false. If $p_{0}, q_{0}$ denote elementary propositions, then, of course, $p_{0} \supset q_{0}$ denotes elementary propositions; but this latter, more complex function denotes a narrower range of propositions than does $t_{0}$, since whatever is an elementary proposition of the form $p_{0} \supset q_{0}$ must be an elementary proposition of the form $t_{0}$, but not conversely. Let $t_{1}$ denote any elementary matrix. Then $t_{1}$ denotes what denotes elementary propositions; $t_{1}$ denotes $t_{0}, p_{0} \supset q_{0}$, and the like, which denote elementary propositions. Now we can form such functions as $p_{1} \supset q_{1}$, which denote a narrower range of matrices than does $t_{1}$.

Since elementary matrices are neither true nor false, we cannot say $\left(t_{1}\right) . t_{1} \vee \sim t_{1}$; but we can say

$$
\left(t_{1}\right):\left(t_{0}\right) . t_{0} \vee \sim t_{0}
$$

\footnotetext{
* Presented to the Society, September 9, 1927.

† See Principia Mathematica, second edition, vol. 1, p. xxiii.
} 
that is, every value of every matrix is true or false. Variables such as $t_{0}, t_{1}$ fall into a hierarchy of values and values of values. We can write such propositions as $\left(t_{1}\right):\left(\exists t_{0}\right) \cdot t_{0}$, that is, some value of every matrix is true (which is false), and $\left(\exists t_{1}\right):\left(t_{0}\right) \cdot t_{0}$, that is, all values of some matrix are true (which is true); and we have, of course,

$$
\begin{aligned}
& \left(t_{1}\right)\left(t_{0}\right) \cdot t_{0} \cdot \vee \cdot\left(\exists t_{1}\right)\left(\exists t_{0}\right) \cdot \sim t_{0}, \\
& \left(t_{1}\right)\left(\exists t_{0}\right) \cdot t_{0} \cdot \vee \cdot\left(\exists t_{1}\right)\left(t_{0}\right) \cdot \sim t_{0},
\end{aligned}
$$

and the like. Consider the proposition

$$
\left(t_{1}\right) .\left(t_{0}\right) t_{0} \vee\left(\exists t_{0}\right) \sim t_{0},
$$

that is, every matrix is such that all of its values are true or at least one of its values is false. This proposition, although a consequence of the proposition $(p) . p \vee \sim p$, cannot be stated without the use of some variable such as $t_{1}$. We have also, of course,

$$
\left(t_{2}\right):\left(t_{1}\right)\left(\exists t_{0}\right) \cdot t_{0} \cdot \vee \cdot\left(\exists t_{1}\right)\left(t_{0}\right) \cdot \sim t_{0},
$$

which is different from

$$
\left(t_{1}\right)\left(\exists t_{0}\right) \cdot t_{0} \cdot \vee \cdot\left(\exists t_{1}\right)\left(t_{0}\right) \cdot \sim t_{0} ;
$$

and it would seem that the proposition

$$
\left(\exists t_{2}\right):\left(t_{1}\right)\left(\exists t_{0}\right) \cdot t_{0} \cdot \vee \cdot\left(\exists t_{1}\right)\left(t_{0}\right) \cdot \sim t_{0}
$$

is not equivalent to any proposition which can be stated without the use of some variable such as $t_{2}$.

3. Values of Functions and Species of Functions. There are two ways in which inferences can be drawn from universal propositions of the kind with which we are concerned: we can replace a universally quantified function by one of the values which it denotes, and we can replace a universally quantified function, as genus, by a more determinate function, as species.* Thus,

$$
\left(t_{1}\right) .\left(\exists t_{0}\right) t_{0} \vee\left(t_{0}\right) \sim t_{0}
$$

* See Principia Mathematica, second edition, vol. I, p. xxiii. 
implies

$$
\exists\left(p_{0} \vee q_{0}\right) . p_{0} \vee q_{0} . \vee .\left(p_{0} \vee q_{0}\right) . \sim p_{0} . \sim q_{0}
$$

that is,

$$
\left(\exists p_{0}, q_{0}\right), p_{0} \vee q_{0}, \vee \cdot\left(p_{0}, q_{0}\right) . \sim p_{0}, \sim q_{0} .
$$

Again,

implies

$$
\left(t_{1}\right)\left(\exists t_{0}\right) . t_{0} \vee \sim t_{0}
$$

$$
\left(p_{1} \vee q_{1}\right) \exists\left(p_{0} \vee q_{0}\right): p_{0} \vee q_{0} . \supset . p_{0} \vee q_{0},
$$

that is,

$$
\left(p_{1}, q_{1}\right)\left(\exists p_{0}, q_{0}\right): p_{0} \vee q_{0} . \supset . p_{0} \vee q_{0} .
$$

It is to be noted that genus and species are to be understood in intension: being a function of the form $p_{1} \vee q_{1}$ entails being a function of the form $t_{1}$; and this is the ground of the implication. There are two ways in which particular propositions can be inferred: we can infer a particular proposition from an instance of the function which is quantified particularly, and we can infer a particular proposition from a more determinate particular proposition. Thus, $\left(t_{0}\right) . t_{0} \vee \sim t_{0}$ implies $\left(\exists t_{1}\right)\left(t_{0}\right) . t_{0}$; and $\exists\left(p_{0}, q_{0}\right) \cdot p_{0} . q_{0}$ implies $\left(\exists t_{0}\right) \cdot t_{0}$.

4. Analysed Propositions. Heretofore we have been concerned with matrices whose values are elementary functions of unanalysed propositions, and we have illustrated the way in which a hierarchy of functions can be formed in this connection. Thus we may have

$$
p_{2} \vee q_{2}
$$

which has as a typical value

$$
p_{1}^{\prime} \cdot p_{1}^{\prime \prime} \cdot v \cdot q_{1}^{\prime} \cdot q_{1}^{\prime \prime},
$$

which has as a typical value

$$
r_{0}^{\prime} \supset s_{0}^{\prime} \cdot r_{0}^{\prime \prime} \supset s_{0}^{\prime \prime} \cdot \boldsymbol{v} \cdot t_{0}^{\prime} \supset u_{0}^{\prime} \cdot t_{0}^{\prime \prime} \supset u_{0}^{\prime \prime},
$$

which has as a value any elementary proposition of this form.* I wish to explain how a similar hierarchy can be formed in connection with analysed propositions. A propo-

* See Principia Mathematica, loc. cit., p. xxxi, etc. 
sition is a value of a function if the function can be obtained by replacing constituents of the proposition by appropriate variables. We are to consider propositions which are values of relational functions, such as the proposition " $a$ gives $b$ to $c . "$ This proposition is a value of each of the functions " $a$ gives $b$ to $x$," $f(a, b, c), f(a), f(x), f(x, y, z)$, among others. Commonly, one and the same proposition is open to various analyses. If we consider an appropriate constituent of a relational proposition, two entities appear which can be replaced by variables, namely this constituent and the remainder of the proposition. It is, however, often difficult to decide what are the constituents of a proposition which can be replaced by variables.

Now a proposition of the form $R a b$ is a value of the function $R a y$; and it is clear that $R a y$ can be obtained from $R x y$ by replacing $x$ by $a$. We may indicate this order of denoting by writing $R x_{1} y_{0}$; so that $R x_{1} y_{0}$ denotes $R a y_{0}$, which denotes $R a b$. Subscripts indicate parameters of various orders, and accordingly they indicate the order of denoting; so that the subscript which attaches to a variable determines the point in the hierarchy at which the variable takes values. Thus a function $f(x, y, z)$ is ambiguous as regards the parametric order of its variable constituents. We may have $f_{1}\left(x_{0}, y_{0}, z_{0}\right)$, which denotes " $x_{0}$ gives $y_{0}$ to $z_{0}$," which denotes " $a$ gives $b$ to $c$ "; or, we may have $f_{0}\left(x_{1}, y_{1}, z_{1}\right)$, which denotes $f_{0}(a, b, c)$, which denotes " $a$ gives $b$ to $c$ "; and so on. It is clear that, as a result of this analysis, we can attach an applicative, "some" or "every," to the entire function at each stage of the hierarchy. If we apply "some" to $f_{1}\left(x_{0}, y_{0}\right.$, $\left.z_{0}\right)$ and "every" to $f_{0}\left(x_{0}, y_{0}, z_{0}\right)$, we have

$$
\exists f_{1}\left(x_{0}, y_{0}, z_{0}\right):\left(f_{0}\left(x_{0}, y_{0}, z_{0}\right)\right) \cdot f_{0}\left(x_{0}, y_{0}, z_{0}\right),
$$

which corresponds to $(\exists f):(x, y, z) . f(x, y, z)$ in the ordinary analysis; whereas, if we apply "some" to $f_{0}\left(x_{1}, y_{1}, z_{1}\right)$ and "every" to $f_{0}\left(x_{0}, y_{0}, z_{0}\right)$, we have

$$
\exists f_{0}\left(x_{1}, y_{1}, z_{1}\right):\left(f_{0}\left(x_{0}, y_{0}, z_{0}\right)\right) . f_{0}\left(x_{0}, y_{0}, z_{0}\right) \text {, }
$$

which corresponds to $(\exists x, y, z):(f) . f(x, y, z)$. 
Now although the proposition $\left(f_{1} x_{0}\right):\left(f_{0} x_{0}\right) \cdot f_{0} x_{0}$ corresponds to $(f):(x) \cdot f x$, there is an important difference between these propositions which I wish to point out. When we say $(f):(x) . f x$, it is presupposed that each value of $x$ can be combined with every value of $f$ to form a significant proposition; whereas, no such presupposition as this is involved when we write $\left(f_{1} x_{0}\right):\left(f_{0} x_{0}\right) \cdot f_{0} x_{0}$. If there should be values of $f_{0}$, say $f^{\prime}, f^{\prime \prime}$, and values of $x_{0}$, say $x^{\prime}, x^{\prime \prime}$, such that $f^{\prime} x^{\prime}$ and $f^{\prime \prime} x^{\prime \prime}$, but such that $f^{\prime} x^{\prime \prime}$ and $f^{\prime \prime} x^{\prime}$ are nonsignificant, this fact would not render $\left(f_{1} x_{0}\right):\left(f_{0} x_{0}\right), f_{0} x_{0}$ either false or non-significant. A matrix is to be regarded as taking such values as exhibit the form of the matrix; and the values taken by a variable constituent of a matrix depend upon the matrix in which the variable occurs. It is therefore possible that $\left(f_{1} x_{0}\right):\left(f_{0} x_{0}\right) \cdot f_{0} x_{0}$ should have a wider reference than $(f):(x) \cdot f x$.

5. Analysed and Unanalysed Propositions. I wish now to consider functions whose values are elementary functions of analysed propositions, and to inquire how, if at all, the denotative hierarchy for elementary functions of unanalysed propositions can be combined with the hierarchy for analysed propositions. We have such propositions as $\left(f_{1} x_{0}\right)\left(\exists f_{0} x_{0}\right) \cdot f_{0} x_{0}$ and $\left(\exists f_{0} x_{1}\right)\left(f_{0} x_{0}\right) \cdot f_{0} x_{0}$. Now it appears that we can extend the range of $t_{0}, t_{1}, \cdots$ so as to include such functions as $f_{0} x_{0}, f_{1} x_{0}, \cdots$. This can be seen to be possible by noting that $f_{0} x_{0}$ is a species of $t_{0}$, and that $f_{1} x_{0}$ is a species of $t_{1}$. An analysed function will have the order of its constituents of highest parametric order. It is clear that, for example, $\left(f_{0} x_{0}\right) \cdot f_{0} x_{0} \vee \sim f_{0} x_{0}$ follows from $\left(t_{0}\right) . t_{0} \vee \sim t_{0}$, and that $\left(f_{0} x_{0}\right) f_{0} x_{0} \vee\left(\exists f_{0} x_{0}\right) \sim f_{0} x_{0}$ follows from $\left(t_{0}\right) . t_{0} \vee\left(\exists t_{0}\right) \sim t_{0}$; and also that $\left(\exists f_{0} x_{0}\right) f_{0} x_{0}$ entails $\left(\exists t_{0}\right) t_{0}$. Consider the proposition

$$
\left(t_{1}\right) .\left(\exists t_{0}\right) t_{0} \vee\left(t_{0}\right) \sim t_{0} .
$$

If we choose $f_{1} x_{0}$ as a species of $t_{1},(a)$ is seen to entail

$$
(f) .(\exists x) f x \vee(x) \sim f x ;
$$


but if we choose $f_{0} x_{1}$ as a species of $t_{1},(a)$ is seen to entail

$$
(x) .(\exists f) f x \vee(f) \sim f x .
$$

Functions of any number of variables can replace $t_{1}$, but, in any case, doubly-quantified propositions result. Of course, we can assign values to $t_{1}$, instead of replacing it by species. We can, for example, substitute $f x_{0}$ (where $f$ is constant), so that we have

$$
(\exists x) f x \vee(x) \sim f x ;
$$

or, if we substitute $f_{0} a$, we have

$$
(\exists f) f a \vee(f) \sim f a .
$$

This analysis gives us a certain advantage as against the ordinary analysis. In the ordinary analysis, we can write $(p) . p \vee \sim p$, and derive, as a species, $(f, x) . f x \vee \sim f x$; but this is confined to singly-quantified propositions, that is, to propositions involving a single applicative, "some" or "every"; whereas, we are now able to write, say, $\left(p_{n}\right) \ldots$ $\left(\exists p_{0}\right) \cdot p_{0} \vee \sim p_{0}$, which is a proposition involving $n+1$ applicatives.

6. Multiply-Quantified Propositions. Let $F\left(t_{0}\right)$ denote an elementary function of $t_{0}$. Then it is clear that $\left(t_{0}\right) . F\left(t_{0}\right)$ implies $\left(t_{1}\right):\left(t_{0}\right) . F\left(t_{0}\right)$. Similarly, $\left(t_{1}\right):\left(t_{0}\right) . F\left(t_{0}\right)$ implies $\left(t_{2}\right): .\left(t_{1}\right):\left(t_{0}\right) \cdot F\left(t_{0}\right)$. Now $\left(\exists t_{0}\right) \cdot F\left(t_{0}\right)$ can be obtained from $\left(t_{0}\right) . F\left(t_{0}\right)$; and generally, in a function of the form $\left(t_{n}\right) \ldots$ $F\left(t_{0}\right)$, we can turn any universal variable into a particular variable. Accordingly, any proposition on $F\left(t_{0}\right)$, of whatever degree of quantification, can be obtained from $\left(t_{0}\right) . F\left(t_{0}\right)$.

In the argument of the last paragraph, we have used $F\left(t_{0}\right)$ to denote an elementary function of $t_{0}$; but I should like to point out that it is not at all necessary to employ such expressions as $F\left(t_{0}\right)$. The proposition $(\exists F):\left(t_{0}\right) . F\left(t_{0}\right)$, for example, expresses what is expressed by $\left(\exists t_{1}\right):\left(t_{0}\right) \cdot t_{0}$. We can express the proposition

$$
(F):\left(t_{0}\right) \cdot F\left(t_{0}\right) \cdot \supset \cdot\left(t_{1}\right)\left(t_{0}\right) \cdot F\left(t_{0}\right)
$$


in the following way. Note that $\left(\exists t_{0}\right) \cdot t_{0}$ is equivalent to $\left(\exists t_{1}\right)\left(\exists t_{0}\right) \cdot t_{0}$; so that we can write

$$
\left(t_{2}\right):\left(\exists t_{1}\right)\left(\exists t_{0}\right) . \sim t_{0} . \vee .\left(t_{1}\right)\left(t_{0}\right) . t_{0} .
$$

Mr. Russell has shown how elementary functions of propositions that are not elementary can be derived from elementary matrices.* Thus, $(x) . f x \vee \sim f x$ implies $(x)(\exists y) . f x$ $\checkmark \sim f y$, which is equivalent to $(x) f x \vee(\exists y) \sim f y$. In precisely the same way,

implies

$$
\left(t_{1}\right)\left(t_{0}\right) . t_{0} \vee \sim t_{0}
$$

$$
\left(t_{1}\right):\left(t_{0}\right)\left(\exists t_{0}^{\prime}\right) . t_{0} \vee \sim t_{0}^{\prime},
$$

which is equivalent to $\left(t_{1}\right) .\left(t_{0}\right) t_{0} \vee\left(\exists t_{0}\right) \sim t_{0}$, which implies

$$
\left(t_{1}\right)\left(\exists u_{1}\right) .\left(u_{0}\right) u_{0} \vee\left(\exists t_{0}\right) \sim t_{0},
$$

which is equivalent to

$$
\left(\exists t_{1}\right)\left(t_{0}\right) t_{0} \vee\left(t_{1}\right)\left(\exists t_{0}\right) \sim t_{0} .
$$

It is clear that elementary functions of propositions of the first and second orders can be derived in this way, whatever the degree of quantification of these propositions. Moreover, from $\left(t_{1}\right) .\left(\exists t_{0}\right) t_{0}$ we derive both $(\exists x) f x$ and $(\exists f) f a$, although one of these latter propositions would be said to be of the first order and the other of the second order. Furthermore, in a proposition such as $\left(t_{0}\right) t_{0}$ there seems to be no reason for restricting $t_{0}$ to elementary propositions; it can denote propositions of the first and second orders as well. Let " $(\exists x) . a$ gives $b$ to $x$ " be denoted by $f(a, b)$. Then

results from

$$
(\exists a, b) f(a, b) \vee(a, b) \sim f(a, b)
$$

$$
\left(t_{1}\right) .\left(\exists t_{0}\right) t_{0} \vee\left(t_{0}\right) \sim t_{0} .
$$

It does not matter that $f$ is not elementary, since it occurs as an unanalysed constituent.

HARVARD UNIVERSITY

\footnotetext{
* See Principia Mathematica, loc. cit., *8.
} 